\title{
Educación antitabáquica en escolares en grupos de riesgos y los índices para su evaluación
}

\section{Toobacco Education School in Risk Groups and Indixes for Evaluation}

\author{
Karel Llopiz Guerra* \\ Universidad Central "Marta Abreu" de Las Villas, Santa Clara, Cuba \\ ORCID: https://orcid.org/0000-0002-1500-8000 \\ Tomas Crespo Borges \\ Universidad Central "Marta Abreu" de Las Villas, Santa Clara, Cuba \\ ORCID: https://orcid.org/0000-0001-6824-8975 \\ Yudith Ivonne Alata Cusy \\ Universidad Nacional Mayor de San Marcos, Lima, Perú \\ ORCID: https://orcid.org/0000-0001-9123-976X \\ Sandy Dorian Isla Alcoser \\ Universidad Nacional Mayor de San Marcos, Lima, Perú \\ ORCID: https://orcid.org/0000-0003-1330-3716 \\ Edgar Froilan Damián Núñez \\ Universidad Nacional Mayor de San Marcos, Lima, Perú \\ ORCID: https://orcid.org/0000-0001-7499-8449
}

Recibido 12-07-18 Revisado 22-07-18 Aprobado 15-09-19 En línea 23-10-19

*Correspondencia

Email: kllopiz@uclv.cu
Citar como:

Llopiz, K., Crespo, T., Alata, Y., Isla, S., \& Damián, E. (2020). Educación antitabáquica en escolares en grupos de riesgos y los índices para su evaluación. Propósitos y Representaciones, 8(2), e403. doi: http://dx.doi.org/10.20511/pyr2020.v8n2.403 


\section{Resumen}

El tabaquismo constituye uno de los flagelos que padece la sociedad contemporánea y los niños no se encuentran exentos ante este riesgo, hecho que se convierte en punto de partida para realizar esta investigación basada en los criterios de educación para la salud y particularmente, en la educación antitabáquica. El objetivo de la investigación busca analizar los conocimientos y los comportamientos obtenidos por un grupo seleccionado de escolares de cuarto grado en grupos de riesgo ante el tabaquismo permitiendo medir los cambios mediante los índices ponderados para su evaluación. Se aplicaron diferentes métodos e instrumentos de nivel empírico como el análisis de documentos, la observación, el análisis de los productos de la actividad, la prueba pedagógica en la etapa inicial y final, donde se organizaron y sistematizaron las fuentes de información en función del objeto de estudio. El análisis y discusión de los resultados en la etapa inicial y final se expresó a través del control y evaluación cuantitativa de las variables en orden con el propósito de garantizar la validez de los resultados, además se analizaron los criterios cualitativos logrados sobre la educación antitabáquica.

Palabras clave: Tabaquismo; Educación antitabáquica; Grupos de riesgo; Escolares; Índices de evaluación.

\section{Summary}

Smoking is one of the scourges suffered by contemporary society, and children are not exempt from this risk, a fact that becomes a starting point for conducting this research based on the criteria of health education and particularly, in the smoking education. The objective of the research seeks to analyze the knowledge and behaviors obtained by a selected group of fourth-grade schoolchildren in risk groups before smoking, allowing the changes to be measured using the weighted indices for their evaluation. Different methods and instruments of empirical level were applied such as document analysis, observation, analysis of the products of the activity, the pedagogical test in the initial and final stage, where the sources of information were organized and systematized according to the object study. The analysis and discussion of the results in the initial and final stage was expressed through the control and quantitative evaluation of the variables in order with the purpose of guaranteeing the validity of the results, in addition the qualitative criteria achieved on smoking control were analyzed.

Keywords: Smoking; Smoking Education; Risk Groups; Schoolchildren; Assessment Rates.

\section{Introducción}

Ante circunstancias adversas, numerosas personas en el mundo han buscado refugio en las drogas, donde el uso y abuso de estas, trae consigo consecuencias negativas para su salud (del Mar Molero-Jurado, del Carmen Pérez-Fuentes, Gázquez-Linares, \& Barragán-Martín, 2017). Según indican las evidencias de estadísticas mundiales, el tabaquismo cobra una vida cada seis segundos, y es una causa de muerte prevenible, seis millones de personas mueren cada año en el planeta a causa de enfermedades asociadas a esta adicción (Barreto Niño, Bonilla Reyes, Calderón Bonilla, Cantillo Avilez, Viña Lopera, \& Quitián-Reyes, 2018). A partir de los elementos abordados en las asambleas internacionales de salud, se concibe la proyección de estrategias para mejorar los estilos de vida, poder prevenir enfermedades evitables y rehabilitar a las personas, siempre que las condiciones lo propicien.

La Organización Mundial de la Salud (OMS), define el tabaquismo como una enfermedad-adicción crónica, no transmisible y adictiva (OMS, 2010) y además el consumo de tabaco en los países en desarrollo constituye un problema de salud pública cuya iniciación generalmente ocurre en la adolescencia, situación que culmina en el hecho que en la adultez más 
de la mitad de sus componentes posee hábito tabáquico (Leal-López, Sánchez-Queija, \& Moreno, 2019).

En la actualidad todas las sociedades se han complejizado y en algunas se admite la presencia, entre sus miembros, de personas consumistas de drogas legales como el tabaco, y por lo tanto se hace necesario desarrollar desde el contexto pedagógico acciones preventivas para atenuar o eliminar los riesgos del consumo desde las edades más tempranas (Bruma Palacios, 2005; Llopiz, 2011). Por tal motivo, es necesario actuar desde una visión educativa que garantice un mejor control, atenuación y solución a este problema, que permita formar una personalidad del estudiante sana, eficiente y responsable.

Un número Índice es una medida estadística que nos permite estudiar los cambios que se producen en una magnitud simple o compleja con respecto al tiempo o al espacio (de la Fuente, 2018), el principal interés de los índices ponderados es el hecho de poder resaltar o atenuar la influencia de las diferentes cantidades de acuerdo con algún criterio externo (UAM, 2017). Conceptualmente se define un número índice como una medida estadística que permite estudiar las fluctuaciones o variaciones de una magnitud o de más de una en relación con el tiempo o el espacio. Los índices más habituales son los que realizan las comparaciones en el tiempo, por lo que algunos especialistas los consideran como series temporales (Crespo, 2005; Arranz, García Serrano, \& Virginia, 2018).

Los números índices constituyen el instrumento básico para sintetizar las estadísticas económicas, de modo que las fórmulas utilizadas permitan expresar y describir, por ejemplo, el crecimiento económico de un país o la tasa de inflación de una economía. Si se utilizan fórmulas diferentes, los resultados difieren y las comparaciones no son válidas. De ahí la importancia de conocer las fórmulas que se utilizan, y que los países y organismos internacionales promuevan prácticas comunes que armonicen y estandaricen las mediciones. (Guillen, Badii, Garza, \& Acuña, 2015; Dorin, Perrotti, \& Goldszier, 2018)

Aunque los índices surgieron para explicar fenómenos económicos y de las llamadas ciencias exactas y naturales, en la actualidad en las ciencias sociales proliferan los índices; por citar solo algunos se tiene el índice de Pearl para medir la eficacia de métodos anticonceptivos en estudios clínicos con incidencia en un problema social de gran importancia en la actualidad (Pelaez Mendoza, 2018), pero existen otros como el índice de desarrollo humano (IDH) elaborado por el Programa de las Naciones Unidas para el Desarrollo en el que toma en consideración la esperanza de vida al nacer, los niveles de educación, entre otros; pero también existen índices de paz global, de desarrollo sostenible, de pobreza, de alfabetización, de paridad de géneros, de percepción de la corrupción, de longevidad, de nivel de vida, de satisfacción de vida, de calidad de vida. (PNUD, 2017).

Entre estos últimos se estudia y analiza la educación antitabáquica, la cual tiene carácter integral, sistémico, multifactorial y proactivo debiendo sustentarse en una tríada que tiene en cuenta el refuerzo de la autoestima, la información adecuada y las habilidades para la vida (Llopiz, 2018).

El IDH resalta que son las personas y sus capacidades el criterio más importante para evaluar el desarrollo de un país, y no solamente el crecimiento económico (PNUD, 2017), por lo cual para el grupo de escolares en grupos de riesgo se debe actuar desde una concepción preventiva ante esta enfermedad-adicción. Más cercano a las ciencias pedagógicas se han propuesto índices para determinar la calidad del empleo de los medios informáticos en la clase y el índice del nivel integral de calidad de aprendizaje (INICA) (Crespo, 2005).

Los números índices en la práctica proporcionan información sobre diferentes aspectos de los logros de una población y, lo que es más importante, no hay necesidad de elegir entre unas y otras. Por el contrario, se complementan entre sí (PNUD, 2010, Alkire \& Santos, 2014, 
como se citó en Burgos Dávila; Cando Ortega, 2016). En este proceso sistémico de recogida de datos y en la obtención de información válida y fiable para cada una de las variables utilizada para emitir juicios de valor acerca del estado de la calidad educativa, los índices juegan un importante papel; así, se habla de índice de asistencia, de calidad de clases, de analfabetismo, etc (Crespo, 2002; Estupiñan, 2012).

Por lo cual el objetivo de la presente investigación se expresa en analizar los conocimientos y los comportamientos obtenidos por un grupo seleccionado de escolares en grupos de riesgo ante el tabaquismo que permiten medir los cambios mediante los índices ponderados para su evaluación.

\section{Método}

Para la planificación de la intervención experimental se partió del análisis de la población utilizada en un pilotaje inicial de 56 escolares, y a partir de las necesidades declaradas se seleccionó un grupo de cuarto grado, haciéndose medibles los análisis y avances desde las diferentes actividades tanto docentes, extradocentes y extraescolares con la muestra seleccionada de 15 escolares en grupos de riesgos ante el tabaquismo.

La evaluación del estado inicial de los escolares en grupos de riesgo ante el tabaquismo, a partir de los conocimientos y el desarrollo de comportamientos en contra del tabaquismo, permitió desde el diagnóstico inicial la evaluación cuantitativa de los indicadores acerca del desarrollo de la educación antitabáquica alcanzado por los escolares.

La evaluación del comportamiento de las dimensiones e indicadores en la aplicación de los métodos e instrumentos utilizados para el diagnóstico inicial de los escolares en grupos de riesgos estuvo dirigida a: la aplicación de los conocimientos en la práctica pedagógica y los comportamientos que asumen ante las diferentes manifestaciones en la escuela y en el lugar donde conviven. Las dimensiones e indicadores propuestos y evaluados fueron los siguientes: 1) Dominio de los conocimientos acerca del tabaquismo, 2) Comportamientos que asume el sujeto acerca de los riesgos ante el tabaquismo.

En la investigación se consideraron los niveles bajo, medio y alto que permitieron medir los cambios producidos antes y después de la aplicación de las actividades. Para la evaluación integradora de los indicadores se determinó que el nivel bajo comprende dos indicadores bajos, el nivel medio comprende tres indicadores medios o más, el nivel alto comprende tres indicadores altos o más.

Los indicadores del desarrollo alcanzado por los escolares en grupos de riesgo ante el tabaquismo se evaluaron de forma sistemática durante el desarrollo de las diferentes formas organizativas en el orden docente, así como extradocentes y extraescolar realizado con ellos. Para medir cómo se comporta la educación antitabáquica se aplicaron diferentes instrumentos validados desde una etapa preliminar. Entre los métodos de nivel empírico se utilizaron el análisis de documentos, con el objetivo de organizar y sistematizar las fuentes de información en función del objeto que se estudia, la observación, el análisis de los productos de la actividad y la prueba pedagógica en la etapa inicial.

Como potencialidades se expresan los aspectos referidos al Modelo de Escuela Primaria con la concepción de un proceso de enseñanza-aprendizaje desarrollador (Rico, 2000). Además, se encuentra el Programa Director de Promoción y Educación para la Salud el cual expresa el eje temático: Educación Antitabáquica, antialcohólica y antidrogas (2008), pero no se evidencia una sistematización de resultados en el orden científico, que declaren las vías de trabajo en relación con esta problemática. 
$\mathrm{Al}$ establecer la relación entre el estudio de los documentos normativos, los resultados de las observaciones a clases y actividades extradocentes y extraescolares y someterlo a la constatación empírica, se evidenció que las insuficiencias influían negativamente en la dirección del proceso, en el aprendizaje y comportamientos de los escolares (Ramírez, 2011). De esta manera se arribó a un grupo de regularidades:

- En los documentos normativos, programas de estudios, planes de clases, y los medios de enseñanza aparecía poco desarrollado un proceder para dar tratamiento a los contenidos del tabaquismo por la vía docente, extradocente y extraescolar.

- El personal docente al dirigir el proceso no promovió el interés y motivación por desarrollar un adecuado trabajo preventivo ante el riesgo del tabaquismo en función del diagnóstico que poseen de sus escolares.

- No se proyectaron tareas de aprendizaje en relación con el problema que se investiga y no se consolida el proceder y uso de los medios para apropiarse de estos conocimientos teniendo posibilidades de realización a partir de los contenidos que se imparten, así como también en los espacios extradocentes y extraescolares.

- No se favorecía de forma consolidada la reflexión, el establecimiento de nexos con la vida social, el intercambio y socialización del conocimiento a partir de sus vivencias relacionadas con el tabaquismo desde su medio familiar y comunitario.

- El incumplimiento en algunos de los escolares con relación a sus deberes establecidos ha provocado una incidencia negativa en el desarrollo de sus personalidades, tanto en los aspectos cognoscitivos como afectivo-motivacionales

- Se necesitaba una mayor sensibilización y preparación desde los espacios que puede crear la escuela con las familias adictas de los escolares y poder contribuir en sus hijos, el desarrollo de la percepción del riesgo ante el tabaquismo.

- No existían consolidadas referencias científicas que permitan medir el diagnóstico y evaluar este tema en las Ciencias Sociales.

Para establecer las pruebas estadísticas a aplicarse, se tuvo en cuenta que se trata de muestras dependientes desde un prexperimento pedagógico (el mismo grupo antes y después), incluyendo los índices y su ponderación para diagnosticar y evaluar la educación antitabáquica en ese grupo etáreo de escolares. Con el control inicial, sistemático y final en la investigación se garantiza la validez de los resultados obtenidos por los escolares en la asimilación de los conocimientos y comportamientos en función de desarrollar la educación antitabáquica.

\section{Resultados}

Mediante la aplicación de los métodos y materiales utilizados se determinó el nivel de desarrollo de la educación antitabáquica, manifestada por los escolares, vías que permitieron corroborar la tolerancia, aceptación y la falta de preparación para el aprendizaje y comportamientos asumidos ante el tabaquismo desde el diagnóstico inicial.

Para realizar la medición propia se utilizó la estadística descriptiva y se hizo necesario determinar un índice a partir de los indicadores de educación antitabáquica que se establecieron para la investigación. El índice que se presenta como resultado práctico en la investigación permite medir el impacto antes y después sobre los cambios ocurridos en la educación antitabáquica adquirida por los escolares.

El mismo es un índice complejo ponderado que utiliza índices simples asignados a los indicadores de la variable dependiente de la investigación pues según Rosembaum y Highland "un número índice es una forma especial de razón utilizada para mostrar cambios durante el periodo" (Nuño-Gutiérrez, Álvarez-Nemegyei \& Madrigal-de León, 2008, p. 23).

A los índices simples $1 ; 2 ; 3 ; 4$ y 5 en correspondencia con los indicadores determinados se le asignaron los niveles y sus mediciones correspondientes: Alto (3), medio (2), bajo (1) según la 
operacionalización descrita anteriormente. Por su parte, el índice 5 se ponderó tomando en cuenta que: La ponderación es sinónimo de engrandecimiento, loa, enaltecimiento, es decir, algún componente del índice se altera por alguna razón intencionada dándole más peso (Crespo,2006), y siguiendo a este autor, esta ponderación debe estar fundamentada por la teoría que va a incorporar al índice que se construye en su proceso de investigación.

En este caso la necesidad de esa ponderación se justifica porque el indicador: Rechazo de forma verbal y extraverbal ante el tabaquismo debe expresar la mayor aspiración para que los escolares desarrollen el inicio de una consolidación en los procesos que forman la educación antitabáquica a partir del sistema de influencias educativas ejercidas al aplicar la propuesta.

La expresión de cálculo del índice buscado responde a la fórmula:

$$
\frac{\left.\left(\sum_{i=1}^{4} I N D_{-i}\right)+3 I N D_{-5}\right)}{21}
$$

Es de notar que la división por 21 expresa la mayor suma posible de los índices simples que componen el índice compuesto que se estudia. Con este índice se obtiene un valor entre cero y uno, correspondiendo a cero una educación antitabáquica nula, y el uno como una Educación antitabáquica óptima.

En la aplicación este índice permite tener un primer acercamiento a la situación que individualmente presenta cada escolar respecto al tabaquismo y el promedio de estos índices, acompañados del correspondiente coeficiente variacional, puede ser un indicador de la situación global del grupo de estudio, a partir del cual se puede ir a la indagación cualitativa de cada escolar y del sistema de influencias que lo rodea.

En el proceso de investigación este índice contribuye a conocer con profundidad la magnitud de los conocimientos y comportamientos que se demuestran por parte de los escolares en función del tabaquismo donde se pueden realizar comparaciones antes y después de aplicadas las actividades en la investigación y tener una visión individual y global del grupo estudiado que puede demostrarse de forma gráfica en posteriores momentos del trabajo.

Para medir y valorar si ha sido significativo el cambio que se ha producido los índices también favorecen este análisis. A partir de los índices se facilita el procesamiento estadístico lo que hubiera sido más difícil sin tener los indicadores agrupados de la forma en que se presentan anteriormente. Por otro lado, se puede apreciar el cambio que se produjo en los escolares antes y después de aplicada la investigación de forma gráfica.

Todos los análisis expresados anteriormente condujeron a la aplicación de los índices en diferentes momentos de la investigación. Una vez aplicados los instrumentos y la triangulación de los métodos en el diagnóstico inicial se constataron que el desarrollo alcanzado por los escolares en grupos de riesgos ante el tabaquismo se mantiene en niveles bajos, y solo en el indicador 1 se observa una tendencia al nivel alto en 2 escolares pues conocen que el tabaquismo es una enfermedad, para un $(13,3 \%)$. Se evalúa que los indicadores, en su mayoría al aplicar este diagnóstico, los índices de evaluación son bajos.

El comportamiento de la aplicación de las comprobaciones de conocimientos e intercambio con los escolares durante esta etapa fueron mostrando que, de 15 escolares, se ubican en un nivel alto 7 para un (46\%), en 3 escolares, los resultados se comportan en un nivel medio, para un (20\%) y los 5 restantes en un nivel bajo para un (33.3. \%), ofreciéndoles a estos una mayor intencionalidad en las acciones. 
Al valorar las vivencias experimentadas por los escolares relacionados con los riesgos ante el tabaquismo se arriba a la conclusión que fueron muy limitadas sus intervenciones, tal es así que 10 de los 15 estudiantes demostraron un nivel bajo (66.67\%) en este sentido y ninguno nivel alto. Como lo demuestra la tabla de índices por escolares antes de la intervención en la figura 1.

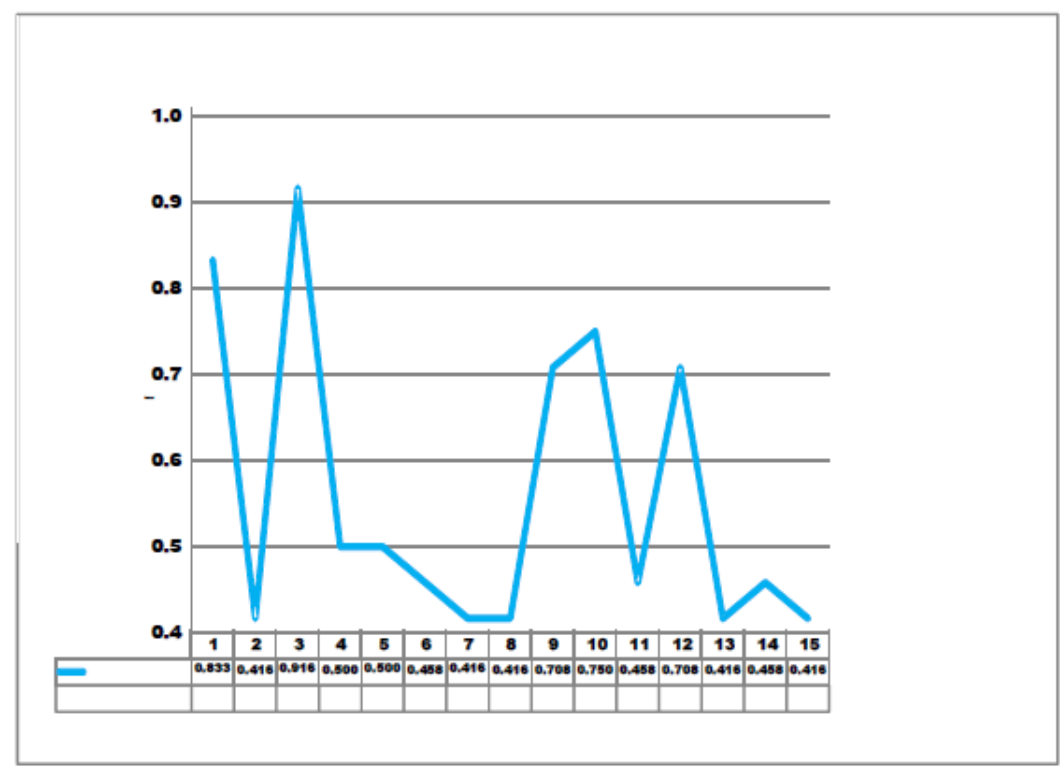

Figura 1. Índices de Educación Antitabáquica de los escolares antes de aplicar el preexperimento pedagógico.

Los resultados que confirmar la necesidad de desarrollar la educación antitabáquica se aprecian inicialmente en los resultados que se obtuvieron en la aplicación de la prueba, las cuales evalúan en sus interrogantes, los aspectos contenidos en cuanto a los conocimientos y comportamientos demostradas relacionadas con la investigación, denotando que no existe una preparación adecuada para combatir esta situación, y una vez más se confirma la relación que existe entre estas dimensiones e indicadores, y su valor preventivo en relación con el tabaquismo. Los instrumentos y las vías de aplicación del diagnóstico por parte de los docentes y el investigador, permitió evaluar la preparación de los escolares en grupos de riesgos ante el tabaquismo y determinar sus necesidades y potencialidades. Los resultados obtenidos indicaron que para obtener éxitos con relación al trabajo preventivo que se desea con los escolares, es necesario desarrollar acciones en función del aspecto docente, extradocentes y extraescolar, que permitieran desarrollar una educación antitabáquica.

Haciendo un análisis del resultado general de la aplicación del diagnóstico, fue posible determinar las necesidades encontradas y corroboradas en la muestra seleccionada con el diagnóstico, se encontraron elementos comunes para propiciar, en función del problema, conocimientos relacionados con el tabaquismo y su nocividad.

A partir de las vivencias afectivas y patrones de imitación ante el consumo, se intencionaron acciones que favorecieron la estimulación y el interés acerca de los daños que provoca al hombre esta adicción, las cuales incidieron en que el escolar comprendiera y estableciera comparaciones entre personas adictas y personas que no consumen cigarros. Estos rasgos les permitieron a los escolares adquirir conocimientos que se ponen de manifiesto en el rechazo de forma verbal y extraverbal a partir de la influencia recibida.

La etapa de comprobación de la investigación se desarrolló mediante la constatación final con el objetivo de evaluar la efectividad de la aplicación de un sistema de actividades docentes, extradocentes y extraescolares vinculadas al tabaquismo y la nocividad de su consumo desde 
edades tempranas en los escolares. Para esta evaluación final se aplicaron diferentes métodos e instrumentos: análisis del producto de la actividad de los escolares, la prueba pedagógica y el completamiento de frases a partir de las vivencias obtenidas.

La aplicación de los instrumentos diseñados con ese fin y el procesamiento estadístico de la información permitieron:

- Valorar las transformaciones en cuanto a los aspectos finales de evaluación en relación con la Educación Antitabáquica alcanzada por los escolares, en comparación con el diagnóstico inicial.

- Valorar la efectividad lograda a partir de la influencia que ejerció en la formación de la personalidad de los escolares.

- Análisis de los resultados de la aplicación en la etapa de comprobación con los escolares en grupos de riesgos ante el tabaquismo.

- En esta etapa se aplicó la prueba pedagógica, que sirvió para evaluar las dos dimensiones declaradas anteriormente, el resultado del análisis del producto de la actividad y el completamiento de frases a los escolares.

Los resultados de los materiales y métodos aplicados expresa desde la dimensión cognitiva, en el indicador 1, el dominio de conocimientos que posee sobre el tabaquismo, donde esta situación cambió favorablemente, ya que 13 escolares, en su mayoría (86.7 \%), llegó a un nivel alto al conocer que el tabaquismo era una enfermedad y una adicción con varios de los argumentos de esas explicaciones; y dos estudiantes mostraron un nivel medio a partir de dominar estas situaciones pero con mayor esfuerzo. Ninguno se mantuvo en el nivel bajo, lo cual se demuestra en la tabla de distribución de frecuencias, y el gráfico de barras que aparecen en la figura 3.

Por último, se refleja que de los dos escolares que al aplicarse el Preexperimento Pedagógico, mostraron un comportamiento medio en el dominio de conocimientos que posee sobre el tabaquismo, uno avanzó de bajo a medio y el otro se mantuvo en medio, además, 8 , que al inicio estaban en un nivel bajo alcanzaron el nivel medio al final y 3 que al inicio estaban en medio pasaron a alto.

En cuanto al indicador 2 expresado en el dominio de conocimientos acerca de los daños que provoca el tabaquismo al final, la mayoría (73.3\%) de los escolares alcanzaron nivel alto al conocer y poder brindar argumentos sobre las enfermedades y consecuencias para la vida que esta adicción provoca al hombre, hasta causar la muerte. Ningún escolar tuvo en este indicador un comportamiento bajo.

Como expresión de la actitud que asume el sujeto acerca del riesgo, al evaluar al final el rechazo expresado de forma verbal y extraverbal ante el tabaquismo, los 15 estudiantes (100\%), rechazan ese consumo a partir de sus manifestaciones expresadas en la prueba pedagógica, que recopila la información en un consenso de evaluar en sus opiniones un marcado rechazo ante el tabaquismo a un nivel alto, por lo que los 10 con nivel bajo, y 3 con nivel medio al inicio alcanzaron, al final, el nivel alto aspectos que se revelan en la figura 2, lo cual demuestra a nivel descriptivo la efectividad de estos indicadores ponderados.

Al tabular los resultados integralmente permitió evaluar el estado final en que el $60 \%$, manifestaron aspectos cognoscitivos logrados y comportamientos de rechazo ante el tabaquismo, donde se reveló un cambio positivo en cuanto al vínculo afectivo ante la atención ofrecida desde las áreas docentes y familiar.

Al hacer un análisis final del producto de la actividad, evaluándose igualmente desde los indicadores en los resultados de las actividades docentes, y las demostraciones en las actividades extradocentes y extraescolares permitió comprobar que, al aplicarse las mismas, hubo un cambio muy favorable en los escolares desde los conocimientos adquiridos y los 
comportamientos de rechazo expresadas en la medida que se involucraron en estas actividades, descubriéndose potencialidades y aptitudes en varias esferas en ellos.

En el gráfico poligonal de la figura 2 aparece el índice ponderado, por escolar, al final de aplicada la investigación a traves del preexperimento pedagogico, donde se observa una vez más el avance de todo el grupo. Este gráfico, refleja el resultado integral de todos los instrumentos aplicados en la investigación, evaluando los indicadores que se han medido durante la aplicación y validación, a partir de los cambios producidos evaluando sus indicadores, ponderando el quinto de ellos, los cuales se muestran en color rojo, donde finalmente quedaron en un nivel alto 9 escolares, para un (60.0\%), y 6 en un nivel medio para un $(40 \%)$, y no quedó ningún escolar en el nivel bajo expresándose en la figura 2.

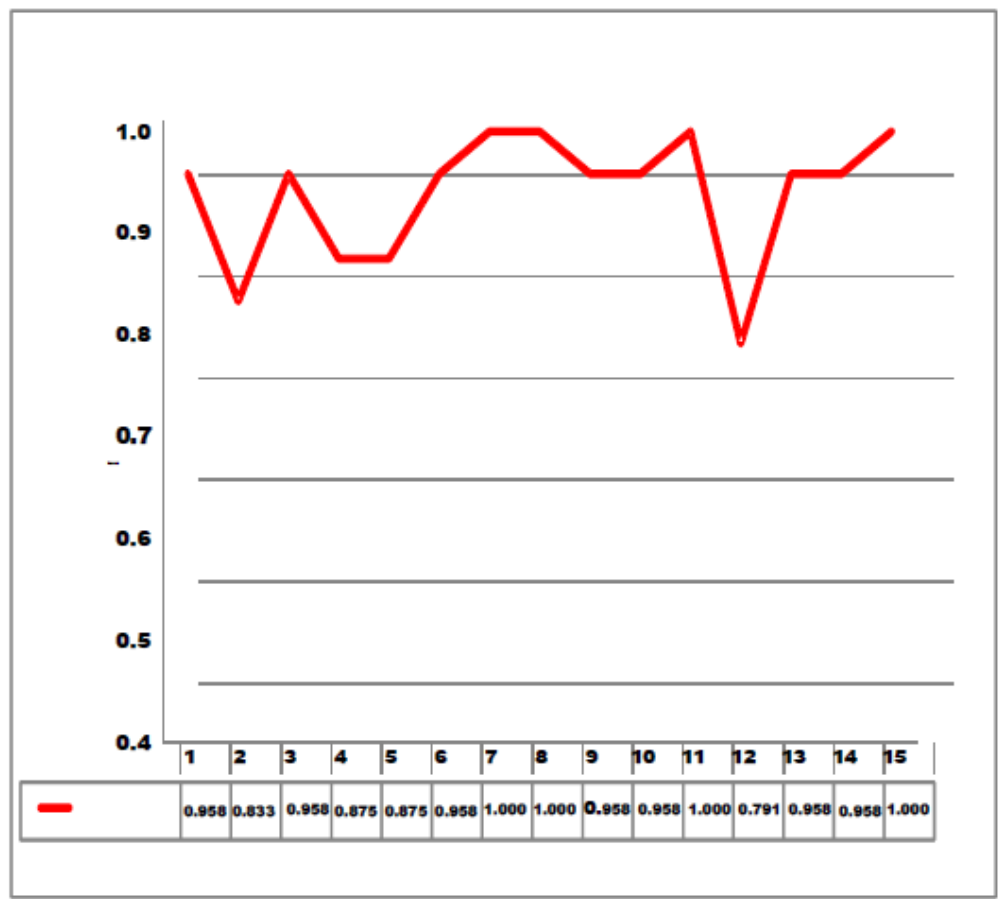

Figura 2. Índices de educación antitabáquica de los escolares al final por índices ponderados al aplicar el preexperimento pedagógico.

Al obtener los resultados antes mencionados se hizo necesario además el cálculo de un índice de cambio relativo con valor máximo de 1 por cada escolar se aprecia seguidamente pues al realizar los análisis de comparación de los resultados antes y después de aplicarse las actividades con los 15 escolares de la muestra, a partir que 12 de ellos (80\%), cambió significativamente por encima de 0.50 y en solo 3 escolares, para un (20\%), los índices de valor se mantuvieron por debajo de 0.50 a partir de mantenerse 2 en un nivel alto y 1 se mantuvo en un nivel medio antes y después, para ello se propone un índice de cambio relativo por alumno cuya expresión matemática es:

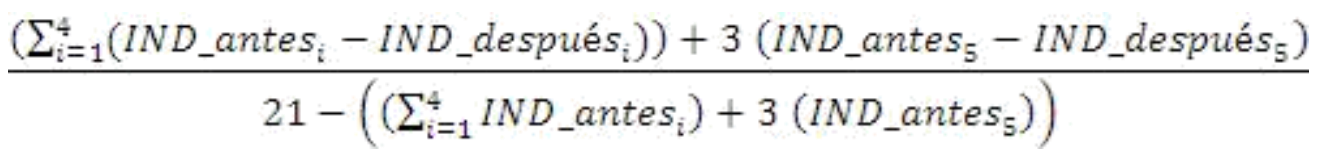

Estos argumentos demuestran una vez más la validez de la aplicación expresado en la figura 3. 


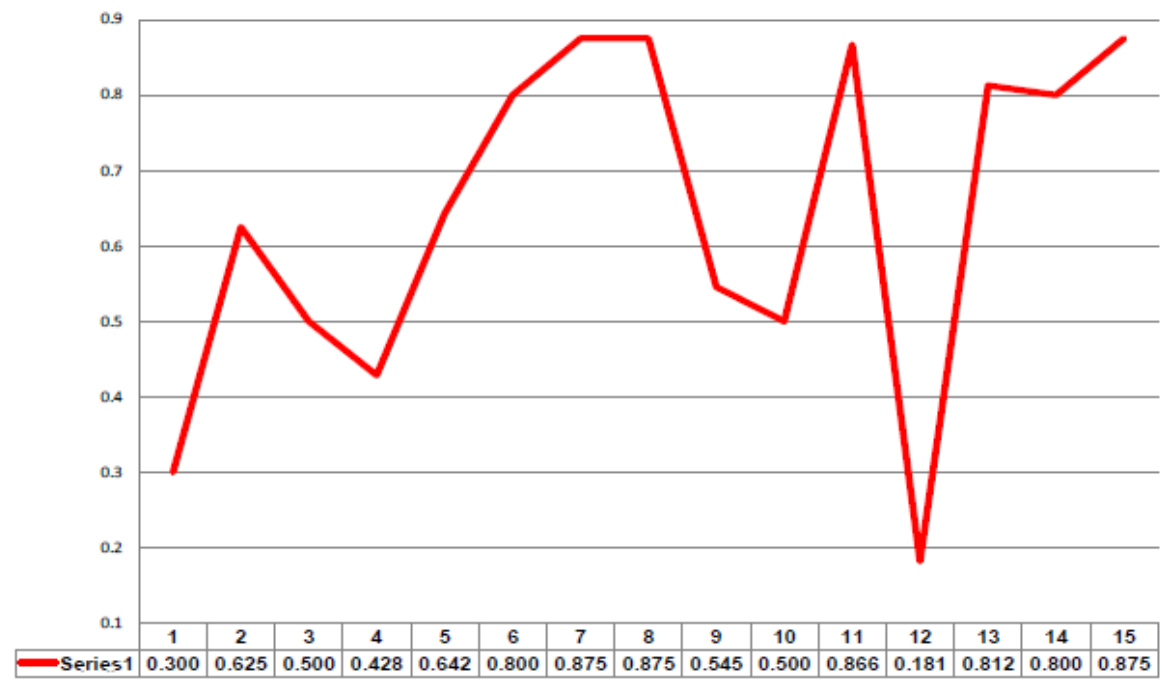

Figura 3. Comparación de los resultados antes y después de aplicarse la investigación mediante un índice de cambio relativo.

Mediante la aplicación de la prueba, los análisis de los productos de las actividades realizadas y el completamiento de frases, después de aplicarse la investigación, permitieron manifestar un cambio positivo con relación al diagnóstico inicial realizado, lo que permitió un rediseño de las acciones que pudieran hacerse donde al triangular los métodos empleados se emplearon los siguientes resultados:

Aunque se realizó la experiencia en un curso escolar y no pueden expresarse de forma consolidada sus resultados de las clases observadas, en su mayoría se cumplieron las exigencias metodológicas en correspondencia con el tipo de actividad, enfocando el trabajo preventivo con los escolares desde el aprendizaje y comportamientos de rechazo ante el consumo, en función de desarrollar una educación antitabáquica ante esta adicción.

Al aplicar a los escolares en el aspecto docente la prueba pedagógica final y el completamiento de frases como complemento para comprobar los conocimientos y comportamientos que demuestren el rechazo que presentan ante el tabaquismo, resultó importante destacar los análisis realizados acerca de los daños que provoca el tabaquismo para sus vidas, denotando un rechazo expresado de forma verbal y extraverbal.

Las actividades extradocentes y extraescolares se planificaron de forma sistemática y en función de los objetivos propuestos, con una mayor vinculación con las clases, donde las motivaciones y el interés por aprender sobre el tema por parte de los escolares fueron creciendo, a medida que las experiencias de los escolares se multiplicaban.

El análisis del producto de la actividad permitió comprobar que, en función de la temática investigada, comenzaron a proyectarse acciones en el orden preventivo que provocó un cambio favorable en todos los implicados.

\section{Discusión}

La educación antitabáquica constituye un proceso donde los escolares pueden apropiarse de un conjunto de saberes conceptuales, procedimentales y del comportamiento sobre el tabaquismo, como una enfermedad adictiva, con un carácter preventivo, siendo un resultado de la labor educativa que realiza la escuela con la familia y la sociedad, para desarrollar un rechazo al 
consumo, contribuyendo al logro del fin y objetivos de la escuela primaria (Varona, Pernas, Socarrás, \& Bancol, 2015).

Al desarrollar actividades que permitieron los momentos iniciales de una educación antitabáquica se expresó un nivel superior en el orden cognoscitivo y del comportamiento, basado en la implementación de índices y la ponderación de uno de ellos en la investigación pedagógica, a partir del rechazo manifestado ante el tabaquismo desde las actividades desarrolladas y la influencia educativa intencionada, logrando un acercamiento al dominio de los conocimientos esenciales acerca de los daños que provoca el tabaquismo, mostrando un alto interés por conocer más aspectos sobre el tema, manifestando cambios positivos en cuanto a las nuevas vivencias obtenidas con evidentes manifestaciones de rechazo en el plano verbal y extraverbal.

\section{Referencias}

Arranz, J., García Serrano, C., \& Virginia, H., (2018). Calidad del empleo: una propuesta de índice y su medición para el periodo 2005-2013. Hacienda Pública Española, 225(2) 133164. Recuperado de https://ideas.repec.org/a/hpe/journl/y2018v225i2p133-164.html

Barreto Niño, A., Bonilla Reyes, P., Calderón Bonilla, J., Cantillo Avilez, M., Viña Lopera, J., \& Quitián-Reyes, H. (2018). Factores asociados al inicio del hábito de fumar en estudiantes universitarios en Bogotá, Colombia. Universitas Medica, 59(2), 17-23. doi: https://dx.doi.org/10.11144/javeriana.umed59-2.ihdf

Bruma Palacios, F. (2005). Factores psicosociales en el consumo de drogas en el adolescente. Inventio, la génesis de la cultura universitaria en Morelos, 1(1), 17-21. Recuperado de http://inventio.uaem.mx/index.php/inventio/article/view/6

Burgos Dávila, S., Cando Ortega, F. (2016). Pobreza multidimensional: índice de Alkire y Foster para Ecuador. Economía, 41(42), 10-52. Recuperado de http://erevistas.saber.ula.ve/index.php/economia/article/viewFile/10416/10354

Carvajal, C. (2002). María Antonia Torres Cueto. La salud escolar y la calidad de la educación. En III Seminario nacional para Educadores: Epígrafe VIII. La Habana.

Carvajal, C., \& Torres Cueto, G. M 2007). Promoción de la salud en la escuela cubana: Teoría y metodología. La Habana: Ed. Pueblo y Educación.

Crespo Hurtado, E., \& Crespo Borges. T. (2002). Los números índices en la evaluación de la Calidad de la educación. Revista Varela. Recuperado de http://revistavarela.uclv.edu.cu/articulos/rv1501.pdf

Cirelda Carvajal, M., \& Torres Cueto, I. (2011). Pedagogía de la promoción de la salud en el ámbito escolar: ¿Una rama de la Pedagogía? La Habana: Ed. Educación Cubana. Curso de Pedagogía Internacional

De la Fuente, S. (2018). Estadística: Números Índices. Universidad Autónoma de Madrid. Recuperado de http://www.estadistica.net/PAU2/numeros-indices.pdf

del Mar Molero-Jurado, M., del Carmen Pérez-Fuentes, M., Gázquez-Linares, J. J., \& BarragánMartín, A. B. (2017). Análisis y perfiles del consumo de drogas en adolescentes: percepción del apoyo familiar y valoración de consecuencias. Atención familiar, 24(2), 56-61. doi: https://doi.org/10.1016/j.af.2017.02.001

Dorin, F., Perrotti, D., \& Goldszier, P. (2018). Los números índices y su relación con la economía. ONU. CEPAL. Recuperado de https://repositorio.cepal.org/bitstream/handle/11362/43974/4/S1700988_es.pdf

Estupiñan, M. A. (2012). Aciertos y desaciertos de la ley antitabáquica en Holguín. Correo Científico Médico de Holguín, 16(4). Recupreado de https://www.medigraphic.com/pdfs/correo/ccm-2012/ccm124r.pdf

Guillen, A., Badii, M. H., Garza, F., \& Acuña, M. (2015). Descripción y Uso de Indicadores de Crecimiento Económico. Revista Daena (International Journal of Good Conscience), 10(1), 138-156. 
Leal-López, E., Sánchez-Queija, I., \& Moreno, C. (2019) Tendencias en el consumo de tabaco adolescente en España (2002-2018). Adicciones 31(4), 289-297. doi: https://doi.org/10.20882/adicciones.1111

LLopiz, K. (2011). El Programa Educativo como herramienta pedagógica para lograr la percepción de riesgo ante el tabaquismo en escolares de la Educación Primaria. Revista Cuadernos de Educación y desarrollo, 3(28). Recuperado de http://www.eumed.net/rev/ced/28/klg2.htm

Llopiz, K. (2018). Educación antitabáquica en escolares primarios desde la labor extensionista universitaria. Edumecentro, 10(3), 71-90.

Márquez Jiménez, A. (2017). Educación y desarrollo en la sociedad del conocimiento. Perfiles educativos, 39(158),3-17. doi: https://doi.org/10.22201/iisue.24486167e.2017.158.58635

Morón. J. (2001). El tabaco como reto educativo. Una revisión desde una perspectiva sociopedagógica. Revista Educación XX1, 4, 125-163. doi: https://doi.org/10.5944/educxx1.4.0.366

Nuño-Gutiérrez, B., Álvarez-Nemegyei, J., \& Madrigal-de León, E. (2008). Efecto de una intervención antitabaco en estudiantes de enseñanza media superior en Guadalajara, México. Salud mental, 31(3), 181-188.

PNUD (2017). El índice de desarrollo humano (IDH). Recuperado de http://hdr.undp.org/sites/default/files/2018_human_development_statistical_update_es.p df

Pelaez Mendoza, J. (2018). El uso de métodos anticonceptivos en la adolescencia. Revista Cubana de Obstetricia y Ginecología, 42(1). Recuperado de http://scielo.sld.cu/scielo.php?script=sci_arttext\&pid=S0138-600X2016000100011

Ramírez, A. (2011). Marco legal contra el tabaquismo: Referente obligado para la lucha antitabáquica. Enfermería universitaria, 8(2), 39-45.

Rico Montero, P. (2008). Modelo de Escuela Primaria cubana: Una propuesta desarrolladora de la educación, enseñanza y aprendizaje. La Habana: Ed. Pueblo y Educación.

Torroella, G. (2002). Aprender a convivir. La Habana: Ed. Pueblo y Educación.

Varona, M., Pernas, I., Socarrás, S., \& Bancol, M. (2015). Estrategia educativa antitabáquica para estudiantes de Tecnología de la Salud de Camagüey. Humanidades Médicas, 15(2), 262282. 